SCIENTIFIC REPORT

\title{
An in vitro study on the compatibility and precipitation of a combination of ciprofloxacin and vancomycin in human vitreous
}

\author{
M Hui, A K H Kwok, C P Pang, S W Cheung, R C Y Chan, D S C Lam, A F B Cheng
}

Br J Ophthalmol 2004;88:218-222

\begin{abstract}
Aims: To investigate the precipitation process of a mixture of vancomycin and ciprofloxacin by equilibrium dialysis and its subsequent effect on the level of available free antibiotics.

Methods: Concentrations of vancomycin and ciprofloxacin in an equilibrium dialysis chamber were measured during the equilibrium process by high performance liquid chromatography and fluorescence polarisation immunoassay. Normal saline (NS), balanced salt solution plus (BSS Plus), and vitreous were used separately as the medium of dialysis.

Results: Precipitation of ciprofloxacin occurred on incubation at $37^{\circ} \mathrm{C}$. It formed precipitate on its own or when mixed with vancomycin in all the three media of NS, BSS Plus, and vitreous. There was more precipitation at higher initial ciprofloxacin concentrations; at $25.0 \mathrm{mg} / \mathrm{l}$ about $75 \%$ free drug in BSS Plus was lost after 72 hours. The extent of precipitation was similar in both NS and BSS Plus. In the dialysis chambers, $20 \mathrm{mg} / \mathrm{l}$ ciprofloxacin dialysed against $125 \mathrm{mg} / \mathrm{l}$ vancomycin was reduced to a concentration about $5.0 \mathrm{mg} / \mathrm{l}$ after 168 hours. Precipitation of vancomycin was negligible. Ciprofloxacin precipitated in vitreous at body temperature, irrespective of the presence of vancomycin. Even after precipitation, the resultant concentration of ciprofloxacin was still higher than the $\mathrm{MIC}_{90}$ of the drug against most Gram negative organisms.

Conclusions: Based on this in vitro study, ciprofloxacin precipitated in vitreous at body temperature, irrespective of the presence of vancomycin or the medium for intravitreal injection. The resultant amount of ciprofloxacin was still higher than the $\mathrm{MIC}_{90}$ of the drug against most Gram negative organisms after precipitation. The authors suggest ciprofloxacin in place of ceftazidime when used in combination with vancomycin for treatment of infective endophthalmitis.
\end{abstract}

1 nfective endophthalmitis is an ophthalmic emergency with the potential of causing blindness. ${ }^{12}$ The usual intravitreal antibiotic treatment includes a combination of vancomycin and aminoglycosides, such as amikacin or gentamicin..$^{3-5}$ Ceftazidime is also frequently administered instead of aminoglycosides, together with vancomycin with acceptable antibacterial efficacy and low toxicity. ${ }^{67}$ However, vancomycin and ceftazidime are incompatible upon mixing, with precipitate formation. ${ }^{8}$ This effect has recently been explored with checkerboard titration and equilibrium dialysis. We showed that ceftazidime precipitated to a significant extent, especially when prepared in balanced salt solution plus (BSS Plus), with resultant free antibiotic concentration being significantly reduced to a level of less than the $\mathrm{MIC}_{90}$ of the drug against many organisms. ${ }^{9}$ Such antibiotic concentration would be inadequate for the treatment of a potentially blinding disease like infective endophthalmitis.

Ciprofloxacin is a fluorinated 4-quinolone; it inhibits the bacterial DNA gyrase activity and disrupts DNA supercoiling, resulting in inhibition of bacteria DNA synthesis and cell death. ${ }^{10}$ Ciprofloxacin has a wide spectrum of anti-Gram negative activities, ${ }^{11}$ and is widely used in various clinical infections, including urinary tract infections, abdominal infections, respiratory tract infections, soft tissue infections, and bone and joint infections. ${ }^{12}$ In rabbit models, no retinal toxicity has been found when ciprofloxacin was injected intravitreally at the therapeutic doses of $0.1-0.5 \mathrm{mg} .{ }^{13}{ }^{14}$ The physiochemical compatibility of ciprofloxacin with several other intravenous agents has also been explored, including normal saline, gentamicin, netilmicin and teicoplanin. Only transient precipitation was observed with teicoplanin, which is also a glycopeptide, as is vancomycin, but not with other agents. ${ }^{15}$

In view of the potential usefulness of ciprofloxacin as an alternative to ceftazidime in the treatment of infective endophthalmitis, we investigated the precipitation process of a mixture of vancomycin and ciprofloxacin by checkerboard titration and equilibrium dialysis in various fluid media.

\section{METHODS}

All experiments were conducted in duplicate, and the mean values taken for analysis. Vitreous was obtained from donor cadaver eyes with prior informed consent. The age of the donors ranged from 65 to 75 years, and the time of preparation was within 1 hour of the death of the donors and stored at $-80^{\circ} \mathrm{C}$ immediately after collection before experimental use as a pool. The degree of syneresis of the pooled vitreous was visually low. Dosages of vancomycin (Abbott, Chicago, IL, USA) and ciprofloxacin (Bayer, Germany) were $1.0 \mathrm{mg}$ and $0.2 \mathrm{mg}$ respectively, prepared in $0.1 \mathrm{ml}$ of $0.9 \%$ normal saline (Otsuka, Guangdong, China) or balance salt solution plus (BSS Plus, Alcon, Fort Worth, TX, USA). The vitreous volume of an adult emmetropic human eye is about $4 \mathrm{ml}$, giving a respective empirical concentration of $0.25 \mathrm{mg} / \mathrm{ml}$ and $0.05 \mathrm{mg} / \mathrm{ml}$ for vancomycin and ciprofloxacin when injected into the vitreous. The actual concentrations would be different, depending on the extent of precipitation and the actual vitreous volume.

\section{Assay of antibiotics}

Ciprofloxacin was assayed by high performance liquid chromatography (HPLC) ${ }^{16}$ and vancomycin by a fluorescence polarisation immunoassay (Abbott TDx, Abbott Laboratories, Diagnostics Division, Abbott Park, IL, USA). 
Table 1 Checkerboard study

\begin{tabular}{lllll}
\hline \multicolumn{4}{l}{ A Initial concentrations (mg/l) of ciprofloxacin/vancomycin prepared in microtitre plate (amount in $\mu \mathrm{g})$} \\
\hline $0 / 0$ & $0 / 250$ & $0 / 125$ & $0 / 62.5$ & $0 / 31.25$ \\
$(0 / 0)$ & $(0 / 125)$ & $(0 / 62.5)$ & $(0 / 31.25)$ & $(0 / 15.625)$ \\
$50 / 0$ & $50 / 250$ & $50 / 125$ & $50 / 62.5$ & $50 / 31.25$ \\
$(25 / 0)$ & $(25 / 125)$ & $(25 / 62.5)$ & $(25 / 31.25)$ & $(25 / 15.625)$ \\
$25 / 0$ & $25 / 250$ & $25 / 125$ & $25 / 62.5$ & $25 / 31.25$ \\
$(12.5 / 0)$ & $(12.5 / 125)$ & $(12.5 / 62.5)$ & $(12.5 / 31.25)$ & $(12.5 / 15.625)$ \\
$12.5 / 0$ & $12.5 / 250$ & $12.5 / 125$ & $12.5 / 62.5$ & $12.5 / 31.25$ \\
$(6.25 / 0)$ & $(6.25 / 125)$ & $(6.25 / 62.5)$ & $(6.25 / 31.25)$ & $(6.25 / 15.625)$ \\
$6.25 / 0$ & $6.25 / 250$ & $6.25 / 125$ & $6.25 / 62.5$ & $6.25 / 31.25$ \\
$(3.125 / 0)$ & $(3.125 / 125)$ & $(3.125 / 62.5)$ & $(3.125 / 31.25)$ & $(3.125 / 15.625)$ \\
\hline B Measurable concentrations (mg/l) of ciprofloxacin/vancomycin after 24 hours of incubation at $37^{\circ} \mathrm{C}$ in NS \\
(calculated amount in $\mu \mathrm{g})$ \\
\hline & & & \\
\hline $0 / 0$ & $0 / 356$ & $0 / 189$ & $0 / 105$ & $0 / 54$ \\
$(0 / 0)$ & $(0 / 107)$ & $(0 / 57)$ & $(0 / 31)$ & $(0 / 16)$ \\
$67 / 0$ & $62 / 376$ & $64 / 184$ & $66 / 78$ & $68 / 56$ \\
$(20 / 0)$ & $(19 / 113)$ & $(19 / 55)$ & $(20 / 23)$ & $(20 / 17)$ \\
$36 / 0$ & $33 / 376$ & $36 / 209$ & $33 / 99$ & $29 / 50$ \\
$(11 / 0)$ & $(10 / 113)$ & $(11 / 63)$ & $(10 / 30)$ & $(9 / 15)$ \\
$15 / 0$ & $13 / 352$ & $13 / 179$ & $14 / 92$ & $14 / 46$ \\
$(4 / 0)$ & $(4 / 105)$ & $(4 / 54)$ & $(4 / 28)$ & $(4 / 14)$ \\
$7 / 0$ & $8 / 570$ & $8 / 261$ & $7 / 131$ & $6 / 52$ \\
$(2 / 0)$ & $(2 / 171)$ & $(2 / 78)$ & $(2 / 40)$ & $(2 / 16)$ \\
\hline
\end{tabular}

C Measurable concentrations $(\mathrm{mg} / \mathrm{l})$ of ciprofloxacin/vancomycin after 72 hours of incubation at $37^{\circ} \mathrm{C}$ in NS (calculated amount in $\mu \underline{\mathrm{g}}$ )

\begin{tabular}{lllll}
\hline $0 / 0$ & $0 / 1288$ & $0 / 637$ & $0 / 249$ & $0 / 42$ \\
$(0 / 0)$ & $(0 / 116)$ & $(0 / 57)$ & $(0 / 25)$ & $(0 / 17)$ \\
$100 / 0$ & $174 / 1180$ & $100 / 345$ & $151 / 238$ & $57 / 50$ \\
$(17 / 0)$ & $(17 / 118)$ & $(15 / 52)$ & $(18 / 29)$ & $(23 / 20)$ \\
$29 / 0$ & $62 / 841$ & $51 / 311$ & $92 / 301$ & $29 / 46$ \\
$(12 / 0)$ & $(9 / 126)$ & $(8 / 47)$ & $(9 / 30)$ & $(12 / 18)$ \\
$31 / 0$ & $43 / 1086$ & $67 / 825$ & $13 / 90$ & $15 / 43$ \\
$(6 / 0)$ & $(5 / 130)$ & $(4 / 50)$ & $(5 / 36)$ & $(6 / 17)$ \\
$5 / 0$ & $5 / 320$ & $6 / 160$ & $6 / 91$ & $6 / 39$ \\
$(2 / 0)$ & $(2 / 128)$ & $(2 / 64)$ & $(2 / 37)$ & $(2 / 16)$
\end{tabular}

D Percentage loss of antibiotics after 24 hours of incubation at $37^{\circ} \mathrm{C}$ in NS based on the amount calculated (ciprofloxacin/vancomycin in \%)

\begin{tabular}{lllll}
\hline $0 / 0$ & $0 / 15$ & $0 / 9$ & $0 / 0 \dagger$ & $0 / 0 \dagger$ \\
$20 / 0$ & $26 / 10$ & $23 / 11$ & $21 / 25$ & $18 / 0 \dagger$ \\
$15 / 0$ & $21 / 10$ & $17 / 0 \dagger$ & $21 / 45$ & $30 / 3$ \\
$30 / 0$ & $38 / 16$ & $38 / 14$ & $33 / 11$ & $38 / 12$ \\
$33 / 0$ & $25 / 0 \dagger$ & $22 / 0 \dagger$ & $30 / 0 \dagger$ & $45 / 0$
\end{tabular}

E Percentage loss of antibiotics after 72 hours of incubation at $37^{\circ} \mathrm{C}$ in NS based on the amount calculated (ciprofloxacin/vancomycin in \%)

\begin{tabular}{lllll}
\hline $0 / 0$ & $0 / 8$ & $0 / 8$ & $0 / 20$ & $0 / 0 \dagger$ \\
$32 / 0$ & $31 / 6$ & $40 / 17$ & $28 / 9$ & $8 / 0 \dagger$ \\
$8 / 0$ & $25 / 0 \dagger$ & $39 / 25$ & $26 / 4$ & $7 / 0 \dagger$ \\
$11 / 0$ & $17 / 0$ & $36 / 21$ & $15 / 0 \dagger$ & $7 / 0 \dagger$ \\
$30 / 0$ & $32 / 0 \dagger$ & $26 / 0 \dagger$ & $25 / 0 \dagger$ & $28 / 1$
\end{tabular}

F Measurable concentrations ( $\mathrm{mg} / \mathrm{l}$ ) of ciprofloxacin/vancomycin after 24 hours of incubation at $37^{\circ} \mathrm{C}$ in BSS Plus (calculated amount in $\mu \mathrm{g}$ )

\begin{tabular}{lllll}
\hline $0 / 0$ & $0 / 270$ & $0 / 138$ & $0 / 63$ & $0 / 33$ \\
$(0 / 0)$ & $(0 / 122)$ & $(0 / 62)$ & $(0 / 28)$ & $(0 / 15)$ \\
$51 / 0$ & $52 / 274$ & $51 / 120$ & $43 / 64$ & $41 / 32$ \\
$(23 / 0)$ & $(24 / 124)$ & $(23 / 54)$ & $(19 / 29)$ & $(19 / 15)$ \\
$28 / 0$ & $27 / 264$ & $28 / 141$ & $27 / 65$ & $28 / 34$ \\
$(13 / 0)$ & $(12 / 119)$ & $(13 / 63)$ & $(12 / 29)$ & $(13 / 15)$ \\
$13 / 0$ & $13 / 269$ & $14 / 147$ & $13 / 64$ & $13 / 33$ \\
$(6 / 0)$ & $(6 / 121)$ & $(6 / 66)$ & $(6 / 29)$ & $(6 / 15)$ \\
$7 / 0$ & $7 / 254$ & $7 / 140$ & $7 / 65$ & $7 / 33$ \\
$(3 / 0)$ & $(3 / 114)$ & $(3 / 63)$ & $(3 / 29)$ & $(3 / 15)$
\end{tabular}

G Measurable concentrations (mg/l) of ciprofloxacin/vancomycin after 72 hours of incubation at $37^{\circ} \mathrm{C}$ in BSS Plus (calculated amount in $\mu \mathrm{g}$ )

\begin{tabular}{lllll}
\hline $0 / 0$ & $0 / 225$ & $0 / 135$ & $0 / 62$ & $0 / 35$ \\
$(0 / 0)$ & $(0 / 101)$ & $(0 / 61)$ & $(0 / 28)$ & $(0 / 16)$ \\
$14 / 0$ & $17 / 245$ & $15 / 137$ & $13 / 67$ & $14 / 34$ \\
$(6 / 0)$ & $(8 / 110)$ & $(7 / 62)$ & $(6 / 30)$ & $(6 / 15)$ \\
$25 / 0$ & $26 / 257$ & $24 / 136$ & $25 / 63$ & $22 / 33$ \\
$(11 / 0)$ & $(12 / 116)$ & $(11 / 61)$ & $(11 / 29)$ & $(10 / 15)$ \\
$14 / 0$ & $13 / 270$ & $13 / 137$ & $13 / 62$ & $13 / 33$ \\
$(6 / 0)$ & $(6 / 121)$ & $(6 / 62)$ & $(6 / 28)$ & $(6 / 15)$ \\
$7 / 0$ & $6 / 256$ & $7 / 135$ & $7 / 62$ & $7 / 33$ \\
$(3 / 0)$ & $(3 / 115)$ & $(3 / 61)$ & $(3 / 28)$ & $(3 / 15)$
\end{tabular}




\begin{tabular}{|c|c|c|c|c|}
\hline \multicolumn{5}{|c|}{$\begin{array}{l}\text { H Percentage loss of antibiotics after } 24 \text { hours of incubation at } 37^{\circ} \mathrm{C} \text { in BSS Plus based on the amount calculated } \\
\text { (ciprofloxacin/vancomycin in \%) }\end{array}$} \\
\hline $0 / 0$ & $0 / 3$ & $0 / 1$ & $0 / 9$ & $0 / 6$ \\
\hline $8 / 0$ & $6 / 1$ & $9 / 14$ & $22 / 7$ & $26 / 7$ \\
\hline $0+/ 0$ & $1 / 5$ & $0+/ 0+$ & $4 / 7$ & $2 / 4$ \\
\hline $4 / 0$ & $4 / 3$ & $1 / 0+$ & $4 / 7$ & $4 / 4$ \\
\hline $2 / 0$ & $6 / 9$ & $1 / 0 \dagger$ & $0 / 6$ & $0+/ 4$ \\
\hline \multicolumn{5}{|c|}{$\begin{array}{l}\text { I Percentage loss of antibiotics after } 72 \text { hours of incubation at } 37^{\circ} \mathrm{C} \text { in BSS Plus based on the amount calculated } \\
\text { (ciprofloxacin/vancomycin in \%) }\end{array}$} \\
\hline $0 / 0$ & $0 / 19$ & $0 / 3$ & $0 / 10$ & $0 / 0$ \\
\hline $75 / 0$ & $69 / 12$ & 73/1 & $78 / 4$ & $76 / 2$ \\
\hline $9 / 0$ & $7 / 7$ & $15 / 2$ & $13 / 9$ & $20 / 5$ \\
\hline $2 / 0$ & $7 / 3$ & $9 / 1$ & $7 / 11$ & $7 / 6$ \\
\hline $5 / 0$ & $8 / 8$ & $0 \dagger / 3$ & $4 / 12$ & $5 / 5$ \\
\hline
\end{tabular}

\section{Study 1: Visual and $\mathrm{pH}$ test}

Standard mixture solutions of $1.0 \mathrm{mg}$ vancomycin and $0.2 \mathrm{mg}$ ciprofloxacin in $0.1 \mathrm{ml}$ of $0.9 \%$ normal saline (NS) or balanced salt solution plus (BSS Plus) were mixed separately with $4.0 \mathrm{ml}$ of NS, BSS Plus, and vitreous for incubation at $37^{\circ} \mathrm{C}$.

\section{Study 2: Checkerboard analysis}

Mixture samples with various concentrations of ciprofloxacin and vancomycin prepared in NS or BSS Plus were incubated at $37^{\circ} \mathrm{C}$ in microtitre plates (table 1) with cover by paraffin foil. Aliquots were taken at 24 hours and 72 hours for assays by HPLC (ciprofloxacin) and TDx (vancomycin) to determine the concentration and amount of free drugs.

\section{Study 3: Equilibrium dialysis in vitreous}

Equilibrium dialysis studies were performed in an equilibrium dialyser (Spectrum Medical Industries, Los Angeles, CA, USA) with a half cell working volume of $1.0 \mathrm{ml}$. Vancomycin $(125 \mu \mathrm{g})$ and ciprofloxacin $(20 \mu \mathrm{g})$ were prepared in NS and added into half cell chamber A which was separated from half cell chamber B by a semipermeable Spectrapor dialysis membrane with a molecular weight cut off at 6000-8000 D (Spectrum Medical Industries). Both chambers were filled with $1.0 \mathrm{ml}$ of vitreous. The whole system was incubated at $37^{\circ} \mathrm{C}$. Aliquots were taken from half cell chamber B at appropriate time intervals up to 168 hours for antibiotic assays. The experiment was then repeated with the antibiotics prepared in BSS Plus and incubated to 192 hours.

\section{RESULTS}

\section{Study 1: Visual and $\mathrm{pH}$ test}

Precipitate was visually detected in NS, BSS Plus, and vitreous after incubation for 1 day at $37^{\circ} \mathrm{C}$. There was no change in $\mathrm{pH}$ before or after precipitation.

\section{Study 2: Checkerboard analysis in microtitre plates}

Vancomycin alone in NS showed no visual precipitation. No measurable decrease was detected at an initial concentration of $31.25 \mathrm{mg} /$ after 24 and 72 hours of incubation. At higher initial concentrations, the measurable loss ranged from $0 \%-$ $20 \%$, median $9 \%$ (first rows, table $1 \mathrm{~B}-\mathrm{E}$ ). Ciprofloxacin alone showed visible precipitation in all wells. Its concentration decreased by $15 \%-33 \%$, median $25 \%$, after 24 hours of incubation, and $8 \%-32 \%$, median $20 \%$, after 72 hours (first columns, table $1 \mathrm{D}$ and $\mathrm{E}$ ).

In mixtures of ciprofloxacin and vancomycin there were similar losses in vancomycin by $0 \%-25 \%$, median $7 \%$, after
24 hours and $0 \%-25 \%$, median $0 \%$, after 72 hours of incubation, suggesting the loss of vancomycin was minimal and independent of ciprofloxacin. Ciprofloxacin loss was $14 \%-45 \%$, median $25 \%$, after 24 hours, and $7 \%-40 \%$, median $26 \%$, by 72 hours, indicating persistent precipitation (table IB-E).

When prepared in BSS Plus, vancomycin alone precipitated from $1 \%-9 \%$, median $4 \%$, after 24 hours. After 72 hours, the loss was $0.03 \%-19 \%$, median $7 \%$ (first row, table $1 \mathrm{H}$ and I), suggesting minimal vancomycin loss. It was independent of whether NS or BSS was used, since the difference in the amount of vancomycin in NS or BSS Plus was insignificant before and after incubation (Student's paired $t$ test). For ciprofloxacin alone, the loss was $0 \%-8 \%$, median 3\%, after 24 hours of incubation. After 72 hours, the decrease was up to $75 \%$ where the initial ciprofloxacin concentration was highest at $25.0 \mathrm{mg} / \mathrm{l}$. At lower initial concentrations ( $12.5 \mathrm{mg} / \mathrm{l}-3.125 \mathrm{mg} / \mathrm{l})$, the ciprofloxacin loss was $2 \%-9 \%$, median 5\%, (first column, table 1I).

In mixture with vancomycin, ciprofloxacin precipitation was $0 \%-26 \%$, median $4 \%$, after 24 hours (table $1 \mathrm{H}$ ). After 72 hours, great loss of ciprofloxacin was observed at an initial high concentration of $25.0 \mathrm{mg} / \mathrm{l}$ (range: $69 \%-78 \%$, median: $74 \%$ ). At lower concentrations, the loss was $0 \%-20 \%$, median $7 \%$ (table $1 \mathrm{I})$.

\section{Study 3: Equilibrium dialysis in vitreous}

First the antibiotics were prepared in NS and injected into dialysis chamber A which was filled with vitreous. The vancomycin concentration in chamber B rose to about $50 \%$ of its original concentration in chamber A after about 24 hours and then plateaued off, showing no loss up to the end of the experiment at 168 hours. For ciprofloxacin, its concentration rose to about $41 \%$ in chamber B by 5 hours, then there was a further small rise in concentration to about $44 \%$ at 24 hours, afterwards it slowly declined to $36 \%$ by the end of the experiment at 168 hours (fig 1A). This suggested a small initial loss of ciprofloxacin by $10 \%$, which slowly increased to about $30 \%$ by 168 hours. The concentrations of ciprofloxacin during equilibrium dialysis were $7.97 \mathrm{mg} / \mathrm{l}$ by 5 hours and $4.94 \mathrm{mg} / \mathrm{l}$ by 168 hours (fig 1B). The duplicate experiment with the antibiotics prepared in BSS Plus showed similar results (fig 2).

\section{DISCUSSION}

Intravitreal injection of combinations of vancomycin and ceftazidime has been advocated and successfully used in the treatment of infective endophthalmitis. ${ }^{5}{ }^{17} 18$ However, in a recent study utilising similar experimental models of check- 

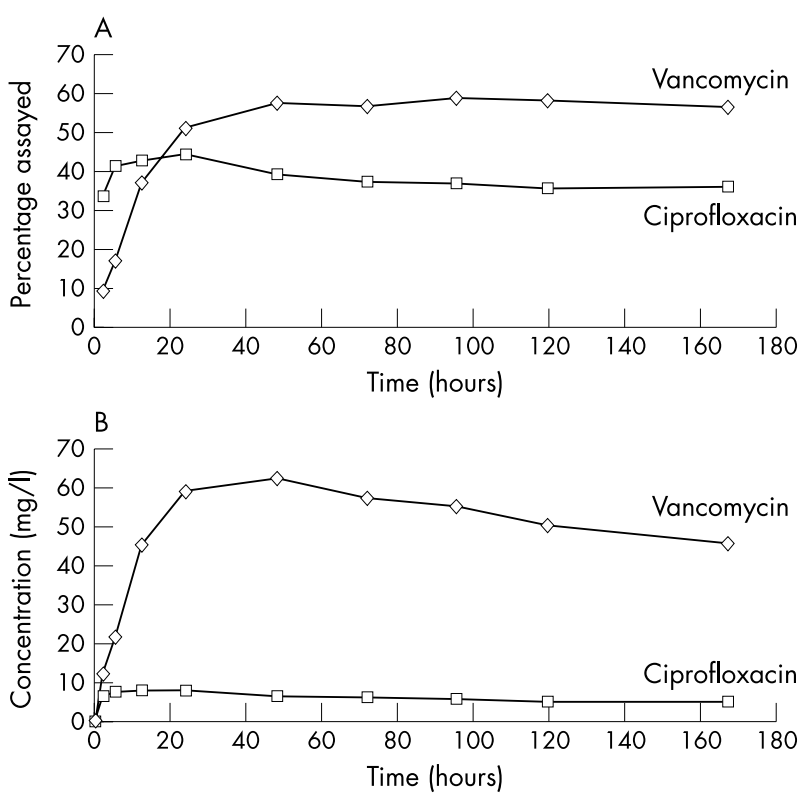

Figure 1 Equilibrium dialysis in vitreous medium with vancomycin and ciprofloxacin initially prepared in normal saline. (A) Percentage change in concentration in vitreous of chamber B. (B) Actual change in concentration in vitreous of chamber $B$.

erboard analysis and equilibrium dialysis as in this present study, we have shown that ceftazidime precipitated on its own or when mixed with vancomycin in any of the three media of NS, BSS Plus, and vitreous. More precipitation was formed if ceftazidime was initially prepared in BSS Plus than in NS, with up to $88 \%$ loss in concentrations of the measurable free antibiotics. ${ }^{9}$

In this present study, we explored the precipitation process of a mixture of vancomycin and ciprofloxacin. In the checkerboard experiments, vancomycin showed negligible to slight loss when prepared alone or in combination with ciprofloxacin. In equilibrium dialysis, the vancomycin origin-
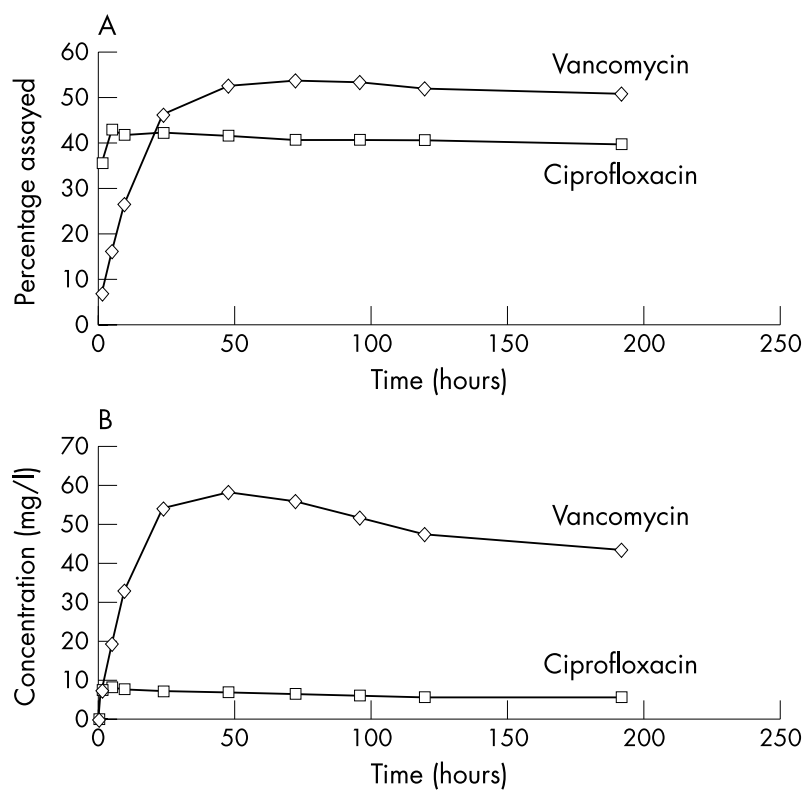

Figure 2 Equilibrium dialysis in vitreous medium with vancomycin and ciprofloxacin initially prepared in balanced salt solution plus. (A) Percentage change in concentration in vitreous of chamber B. (B) Actual change in concentration in vitreous of chamber $B$. ally in chamber A only, became almost evenly distributed in both chambers A and B after incubation to 192 hours. These findings were similar to the results of the equilibrium dialysis process of combinations of vancomycin and ceftazidime. ${ }^{9}$ On the other hand, ciprofloxacin precipitated out irrespective of the presence of vancomycin (range: $20.25 \%-26.3 \%$ ) and the medium of preparation (whether NS or BSS Plus). The precipitation process was stable till 72 hours of incubation. However, the precipitation was greatly enhanced when the initial concentration was at the highest level of $25.0 \mathrm{mg} / \mathrm{l}$ after 72 hours of incubation, suggesting that the ciprofloxacin precipitation was related to high concentrations, and the precipitation process continued on prolonged incubation. The findings of this experimental model have an impact on the design of the dosing regimen in clinical practice. On the one hand, the dosage administered must be high enough to reliably eradicate the offending bacteria; on the other hand, too high a concentration will accelerate its precipitation process, leading to a loss of antibiotic content up to about $74 \%$. Meanwhile, the therapeutic level recommended gives an intravitreal level that is not too high and is at the "supraprecipitation" level. ${ }^{13}{ }^{14}$

Further, in the dialysis experiments, the ciprofloxacin level was decreased from its initial concentration of $20 \mathrm{mg} / \mathrm{l}$ to $7.97 \mathrm{mg} / \mathrm{l}$ by 5 hours and $4.94 \mathrm{mg} / \mathrm{l}$ by 168 hours, no matter whether the antibiotic was prepared in NS or BSS Plus. The antibiotic distributed itself readily in the two dialysis chambers. If there is no loss, the concentration should be $10 \mathrm{mg} / \mathrm{l}$; therefore its level has been lowered by $30 \%$ after 168 hours of dialysis. This is in contrast with ceftazidime in a previous study, in which a loss of $80 \%$ when prepared in NS and of $90 \%$ when prepared in BSS Plus was recorded. ${ }^{9}$ In addition, the remaining ciprofloxacin concentration was many times above the $\mathrm{MIC}_{90}$ of the drug against the common Gram negative bacteria encountered (table 2). ${ }^{19}$ Benz et al have shown that $92 \%$ of Gram negative organisms in culture proved endophthalmitis were susceptible to ciprofloxacin. ${ }^{20}$ In postoperative endophthalmitis, Kunimoto et al have shown that Gram negative isolates were most susceptible to ciprofloxacin $(87.5 \%)$ but were poorly susceptible to ceftazidime $(60.9 \%) .{ }^{21}$ In post-traumatic endophthalmitis, they have also shown that $100 \%$ and $66.7 \%$ of Gram negative organisms were susceptible to ciprofloxacin and ceftazidime, respectively. ${ }^{22}$

In summary, ciprofloxacin precipitates in vitreous, but to a much lesser extent than ceftazidime. This process is independent of the medium, so there is no need to avoid the use of BSS Plus during preparation of the antibiotics nor during intraocular surgery like pars plana vitrectomy. ${ }^{23}$ Vancomycin and ciprofloxacin in a mixture is stable since precipitation is mild. Such stability not only provides an alternative treatment regimen for infective endophthalmitis, but also may avoid frequently repeated intravitreal injections

Table 2 Minimal inhibitory concentration (MIC) of ciprofloxacin against common Gram negative bacteria isolated from the bloodstream in a teaching hospital ${ }^{19}$

\begin{tabular}{lc}
\hline Bacterial species & MIC $_{\mathbf{9 0}}$ (mg/l) \\
\hline Escherichia coli & $\leqslant 0.03$ \\
Klebsiella spp & 0.12 \\
Indole positive Proteus spp & 0.25 \\
Proteus mirabilis & 0.06 \\
Salmonella spp & 0.03 \\
Enterobacter, Citrobacter & 0.25 \\
Acinetobacter spp & 1 \\
Pseudomonas aeruginosa & 0.25 \\
Other non-fermenters & 2 \\
\hline
\end{tabular}


compared with less stable agents. Dosages of vancomycin and ciprofloxacin at $1.0 \mathrm{mg}$ and $0.2 \mathrm{mg}$ respectively, prepared in $0.1 \mathrm{ml}$ of $0.9 \%$ normal saline or BSS Plus, are recommended. Meanwhile, a toxicity study should be conducted to ascertain whether ciprofloxacin poses toxicity effects to the human eye.

\section{Authors' affiliations}

M Hui, S W Cheung, R C Y Chan, A F B Cheng, Department of Microbiology, The Chinese University of Hong Kong, Hong Kong

A K H Kwok, C P Pang, D S C Lam, Department of Ophthalmology and Visual Sciences, The Chinese University of Hong Kong, Hong Kong

A K H Kwok, Department of Ophthalmology, The Hong Kong Sanatorium and Hospital, The Chinese University of Hong Kong, Hong Kong

Financial support: Supported by a block grant, the Chinese University of Hong Kong.

Correspondence to: Dr Alvin K H Kwok, Department of Ophthalmology, Hong Kong Sanatorium and Hospital, 2 Village Road, Happy Valley, Hong Kong; alvinkwok@hksh.com

Accepted for publication 11 March 2003

\section{REFERENCES}

1 Callegan MC, Engelbert M, Parke DW 2nd, et al. Bacterial endophthalmitis: epidemiology, therapeutics, and bacterium-host interactions, Clin Microbiol $\operatorname{Rev} 2002 ; 15: 111-24$.

2 Roth DB, Flynn HW Jr. Antibiotic selection in the treatment of endophthalmitis: the significance of drug combinations and synergy. Surv Ophthalmol 1997:41:395-401.

3 Endophthalmitis Vitrectomy Study Group. Results of the Endophthalmitis Vitrectomy Study. A randomized trial of immediate vitrectomy and of intravenous antibiotics for the treatment of postoperative bacterial endophthalmitis. Arch Ophthalmol 1995;113:1479-96.

4 Campochiaro PA, Lim Jl. Aminoglycoside toxicity in the treatment of endophthalmitis. The Aminoglycoside Toxicity Study Group. Arch Ophthalmol 1994; 112:48-53.

5 Aaberg TM, Flynn HW, Murray TG. Intraocular ceftazidime as an alternative to the aminoglycosides in the treatment of endophthalmitis. Arch Ophthalmol 1994;112:18-19.
$6 \operatorname{Lim} \mathrm{Jl}$, Campochiaro PA. Successful treatment of gram-negative endophthalmitis with intravitreous ceftazidime. Arch Ophthalmol 1992;110:1686.

7 Campochiaro PA, Green WR. Toxicity of intravitreous ceftazidime in primate retina. Arch Ophthalmol 1992;110:1625-9.

8 Lifshitz T, Lapid-Gortzak R, Finkelman Y, et al. Vancomycin and ceftazidime incompatibility upon intravitreal injection. $\mathrm{Br} J$ Ophthalmol 2000;84:117-118.

9 Kwok AKH, Hui M, Pang CP, et al. An in vitro Study of ceftazidime and vancomycin concentrations in various fluid media: implications for use in treating endophthalmitis. Invest Ophthalmol Vis Sci 2002;43:1 182-8.

10 Hooper DC, Wolfson JS. Mode of action of the quinolone antimicrobial agents: review of recent information. Rev Infect Dis 1989;11:S902-11.

11 Eliopoulos GM, Gardella A, Moellering RC Jr. In vitro activity of ciprofloxacin, a new carboxyquinoline antimicrobial agent. Antimicrob Agents Chemother 1984;25:331-5.

12 Walker RC. The fluoroquinolones. Mayo Clin Proc 1999;74:1030-7.

13 Wiechens B, Grammer JB, Johannsen U, et al. Experimental intravitreal application of ciprofloxacin in rabbits. Ophthalmologica 1999;213:120-8

14 Hainsworth DP, Conklin JD, Bierly JR, et al. Intravitreal delivery of ciprofloxacin. J Ocul Pharmacol Ther 1996;12:183-91.

$15 \mathrm{Jim}$ LK. Physical and chemical compatibility of intravenous ciprofloxacin with other drugs. Ann Pharmacother 1993;27:704-7.

16 Chan CY, Lam A, French GL. Rapid HPLC assay of fluoroquinolones in clinical specimens. J Antimicrob Chemother 1989;23:597-604.

17 Han DP, Wisniewski SR, Wilson LA. Spectrum and susceptibilities of microbiologic isolates in the Endophthalmitis Vitrectomy Study. Am J Ophthalmol 1996:122:1-17.

18 Irvine WD, Flynn HW, Miller D, et al. Endophthalmitis caused by gramnegative organisms. Arch Ophthalmol 1992;110:1450-4.

19 Ling TK, Liu EY, Cheng AF. A 13-year study of antimicrobial susceptibility of common gram-negative bacteria isolated from the bloodstream in a teaching hospital. Chemotherapy 2001;47:29-38.

20 Benz MS, Scott IU, Flynn HW, et al. In vitro susceptibilities to antimicrobials of pathogens isolated from the vitreous cavity of patients with endophthalmitis. Invest Ophthalmol Vis Sci 2002;43:E-Abstract 4428.

21 Kunimoto DY, Das T, Sharma S, et al. Microbiologic spectrum and susuceptibility of isolates: Part I. Postoperative endophthalmitis. Am J Ophthalmol 1999;128:240-2.

22 Kunimoto DY, Das T, Sharma S, et al. Microbiologic soectrum and susuceptibility of isolates: Part II. Posttraumatic endophthalmitis. Am J Ophthalmol 1999;128:242-4

23 Moorhead LC, Redburn DA, Merritt J, et al. The effects of intravitreal irrigation during vitrectomy on the electroretinogram. Am J Ophthalmol 1979:88:239-45

24 Edelhauser HF, Van Horn DL, Aaberg TM. Intraocular irrigating solutions and their use for vitrectomy. Monograph 1976;2:265-87. 